\title{
The effect of storage conditions on the deformation patterns of acrylic partial denture bases
}

SADJ March 2020, Vol. 75 No. 2 p72 - p78

\author{
M Motala', A Vahed²
}

\begin{abstract}
Introduction

Incorrect impression-taking techniques and processing errors are known factors contributing to ill-fitting acrylic partial dentures (APDs). Environmental factors such as high temperature and humidity levels can further contribute to the misfits of APDs.

In South Africa, the average summer temperature and humidity level is $21^{\circ} \mathrm{C}$ and $50 \%$, respectively, which may adversely affect APDs during storage postmanufacture.
\end{abstract}

\section{Aim}

To investigate the effect of storage conditions on the deformation patterns of acrylic partial denture bases.

\section{Methods}

Thirty maxillary APD bases were fabricated, and divided into three sample groups, namely: the wet-stored 9-hour cure, dry-stored 9-hour cure, and dry-stored $1 \frac{1}{2}$-hour cure. The dimensional fit in the areas of the incisive papilla, maxillary first molars, and the deepest part of the midline posteriorly were measured using a Mitutoyo ${ }^{\mathrm{TM}}$ micro-meter.

\section{Results}

There were no significant differences in the deformation patterns in the incisal area $(p>0.42)$. Significant differences in the deformation patterns in the maxillary first molar areas $(p<0.04)$ were recorded. The deformation of fit increased as storage time increased.

\section{Author affiliations:}

1. Mohammed Motala: MHSc., Dental Technician, APEX Dental Laboratory, Umhlanga Medical Centre, 321 Umhlanga Rocks Drive, Umhlanga, Durban, South Africa. ORCID Number: 0000-0003-1719-6655

2. Anisa Vahed: D Tech, Senior Lecturer, Dental Sciences Department, Dental Technology Programme, Durban University of Technology, Durban, South Africa.

ORCID Number: 0000-0002-0164-9114

Corresponding author: Mohammed Motala

Dental Technician, APEX Dental Laboratory, Umhlanga Medical Centre, 321 Umhlanga Rocks Drive, Umhlanga, Durban, South Africa.

Email: motalam6@gmail.com

Author contributions:

1. Mohammed Motala: Conception and Design; data collection and analysis; drafting of paper - $50 \%$

2. Anisa Vahed: Data analysis and interpretation; drafting and critically revising of paper - $50 \%$

\section{ACRONYMS \\ APDs: $\quad$ Acrylic Partial Dentures \\ PMMA: Polymerised Polymethyl Methacrylate}

\section{Conclusion}

This study confirms that the deformation patterns of APDs are reduced if kept hydrated on storage.

\section{INTRODUCTION}

Removable acrylic complete and partial dentures are often selected by patients because of the low cost and acceptable aesthetics. ${ }^{1-4}$ Heat-polymerised polymethyl methacrylate (PMMA), commonly referred to as denture base acrylics, is frequently used to fabricate complete and partial dentures. ${ }^{5}$

A known weakness of PMMA as a denture material is that it is porous in nature. Consequently, water, saliva or any other oral fluids can be absorbed during intraoral use and on storage. This can severely compromise the fit, comfort and stability of the denture base in a patient's mouth.

Acrylic dentures that are not stored in water during periods of disuse can deform and become brittle. . $^{2,4,6}$ Deformation of acrylic denture bases stored post-manufacture may also occur in the absence of hydration.

Consani, Monterio and Mesquita et al. ${ }^{1}$ reported on the increased distortion of complete denture bases at room temperature over a six-month dry-storage period.

Consistent with them, Kasina, Ajaz, Attili et al. ${ }^{2}$ demonstrated positive correlations do exist between the polymerisation cycles and degree of hydration and dehydration over a 4-week storage period, which resulted in denture misfits.

This was also evident in Lim and Lee's ${ }^{3}$ recent study, which showed significant linear bilateral posterior buccal flange deformation of complete dentures over a 3-month dry-storage period.

Similarly, South African dental clinicians (Dr Khan and Dr Mohamed, personal communication, March 27, 2015), particularly those who are located in arid areas, have 
observed that the dimensional stability of complete and partial dentures were affected over a two-week dry storage period. The above clinicians alluded that distortion of APDs increased with high temperature and low humidity levels.

In particular, the deformation of the ' $n$ ' shaped maxillary APDs (Figure 1) were greater compared to the ' $w$ ' shaped complete dentures (Figure 2). A point that deserves to be mentioned is that within the context of this study, ' $n$ ' and ' $w$ ' shapes were used to characterise the deformation patterns between an APD and a complete denture, respectively. Notably, deformation of APDs (' $n$ ' shaped) are more likely to be different because of the absence of a buccal flange, which is evident in complete dentures ('w' shaped).

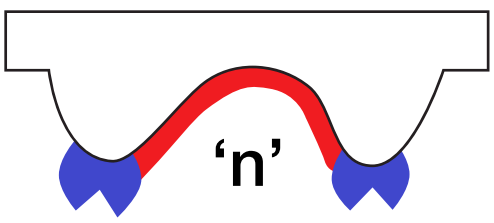

Figure 1. The ' $n$ ' shape of a maxillary APD when viewed from the posterior

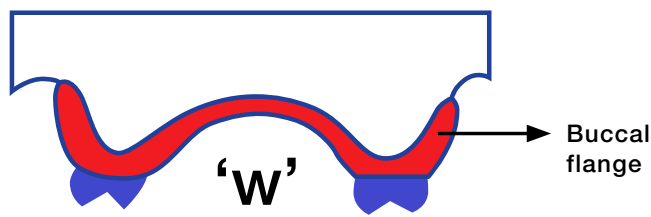

Figure 2. The 'w' shape of maxillary complete denture base when viewed from the posterior.

Despite several studies ${ }^{4-9}$ reporting on the effect of hydration and dehydration of complete acrylic denture bases with varying results, there is limited evidence on the dehydration and deformation of APDs during storage.

This study aims to analyse the effects of dry- and wet-storage conditions on the deformation patterns of ' $n$ ' shape maxillary APD bases. It is anticipated that the results of this study could provide valuable baseline data to dental clinicians in terms of advising patients on denture care and management of APDs during storage.

The hypothesis tested was that there is a significant relationship between dry-storage conditions and deformation of acrylic partial denture bases.

\section{MATERIAL AND METHODS}

Preparation of the denture base specimens

A maxillary cast (Figure 3) was duplicated using silicone moulds (Mold Max ${ }^{\circledR} 30$ Smooth-On, Inc. Macungie, East Texas, USA). These moulds facilitated the duplication of 30 yellow stone models (gypsum calcium sulphate $\alpha$-hemihydrate, Dentsone ${ }^{\mathrm{TM}} \mathrm{KD}$, Saint Gobain, Courbevoie, France).

There were three sample groups, namely: the wetstored 9-hour cure (Control Group); dry-stored 9-hour cure (Group 1); and dry-stored 11/2-hour cure (Group 2). Each sample group had 10 specimens $(n=30)$.
Using the palate area of the model, a maxillary acrylic base plate was subsequently fabricated (Vertex ${ }^{\mathrm{TM}}$ Orthoplast Dental B.V, Netherlands). The acrylic base plate was used as a template (Figure 4) to outline the design on 30 models (Figure 3 ) prior to fabricating the 30 wax pattern specimens (Kemdent $\AA$, Purton, Swindon, Wiltshire, United Kingdom).

All wax pattern were polymerized, according to the manufacturer's recommendations (VerteX ${ }^{{ }^{T M}}$ - Dental B.V, Netherlands). It must be noted that the name assigned to each sample group indicates the type of curing method used to fabricate the APD base specimens. All APD base specimens were trimmed, pumiced and polished to $2 \mathrm{~mm}$ thickness with a high surface lustre.

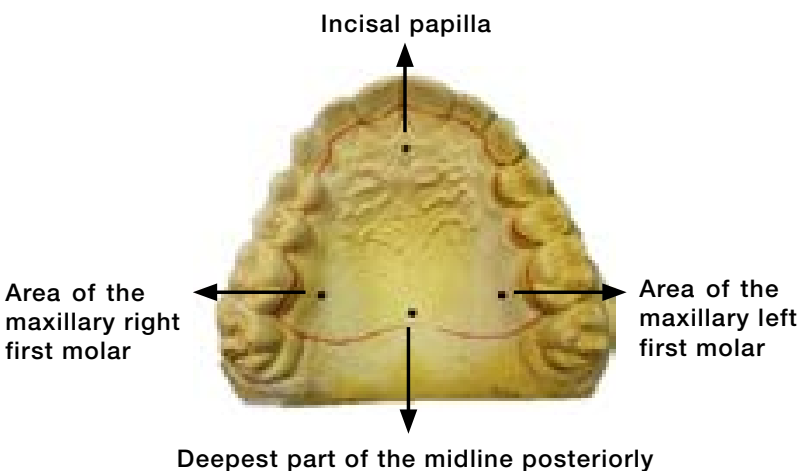

Figure 3. Reference areas on maxillary cast used to gauge the degree of deformation.

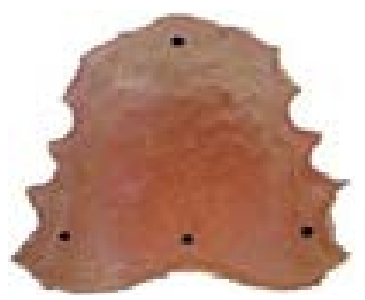

Figure 4. Acrylic base plate used as a template to outline the design on 30 models.

\section{Storage of the specimens}

In determining the extent of deformation of the APD base specimens by temperature and humidity, the dry-stored 9-hour cure and dry-stored 11/2-hour cure were stored in a custom-made incubator for 28 days. The incubator, which was constructed using chipboard $(45 \mathrm{~cm} \times 35 \mathrm{~cm} \times 30 \mathrm{~cm})$, consisted of a fan (Cooler Bank $^{T M}$, Chiefly Choice Limited Company, Shenzhenshi, Canton, China) to cool the air and two 100w light bulbs to increase the temperature within the incubator.

The Relative Humidity $(\mathrm{RH})$ within the unit was controlled using $245 \mathrm{~g}$ of silica gels (Mystify ${ }^{\circledR}$ dehumidifier, Shoprite Checkers Limited, Brackenfell, South Africa). Using a thermostat (Carel ${ }^{\mathrm{TM}}$ s90 Tp1, Padova, Italy) the temperature in the unit was regulated to $21^{\circ} \mathrm{C}$ and kept at $40 \%$ humidity. It must be noted that the temperature and humidity levels of the various South African regions were considered, however the average temperature and humidity levels of arid regions with lower humidity were used. ${ }^{10}$ 
A Thermo-Hygrometer (Flus $®$ FL-201, Shenzhen Flus $®$ Technology, China) was also used to monitor the relative humidity within the unit.

The wet-stored 9-hour cure APD base specimens were stored in a water bath (Mestra ${ }^{\circledR} \mathrm{M}-18$, Talleres Mestraitua S.L. Spain) at $37^{\circ} \mathrm{C}$ for 28 days. The conditions of the experiment were monitored daily and the micro meter was calibrated at the beginning of every storage interval.

\section{Measuring the fit of the acrylic partial} denture base specimens

The degree of the deformations of the APD base specimens were measured following the silicone wafer method used by Ghani et al. ${ }^{11}$ The process entailed mixing $100 \mathrm{~g}$ of Part A Silicone (Mold Max $® 30$; Smooth-On, Inc. Macungie, East Texas, USA) to $10 \mathrm{~g}$ of Part B liquid (Fast cat $\AA$; Smooth-On, Inc. Macungie, East Texas, USA) for three minutes.

The mixture was then poured onto the palatal surface of the maxillary cast and the APD base specimens were fitted onto the cast using finger pressure. Subsequently, a scalpel (Size 23 stainless steel; Omnisurge, Meadowdale Johannesburg, South Africa) was used to trim the excess silicone and the APD base specimens were carefully removed from the cast.

The thickness of the silicone layer was measured in millimetres using a micrometer (Mitutoyo ${ }^{\circledR}$ S293, America Corporation, Illinois, United States of America) at four reference areas.

As illustrated in Figure 3, these reference areas were the incisive papilla, maxillary first molars (16 and 26), and the deepest part of the midline posteriorly.

The fit of the APD base specimens were measured immediately after fabrication (day 1) and subsequently on days $2,7,14,21$ and 28 of the storage period. Consequently, the degree of deformation of the three APD sample groups was recorded.

\section{Statistical analysis}

Using Graph Pad® Prism Version 5 (GraphPad Software Inc. California, USA) a slope test was used to compare the deformation trends of each sample group $(p=0.05)$.

As outlined by Motulsky ${ }^{12}$, laboratory and clinical researchers frequently used Graph Pad® Prism nonlinear regression analysis to fit a straight-line model, and to test whether the best-fit value of the slope differs significantly from 1.0 (or any other value) with 90\% confidence limits.

A noteworthy point is that while the $r^{2}$ provides an estimate of the strength of the relationship between the observed values and the predicted values, it does not provide a formal hypothesis for this relationship.

The F-test $(p<0.05)$ determines whether this relationship is statistically significant.

\section{RESULTS}

The degree of deformation of the APDs, specifically at the four reference areas is outlined below.

\section{Incisal area of the acrylic partial denture base}

As shown in Table 1, the slope test results of the incisal area of the wet-stored (28 days) APD bases revealed a low $r^{2}$ value $(0.001452)$. In contrast to the low $r^{2}$ value of the wet-stored APD bases, the slope test results of the incisal region of the dry-stored APD bases revealed high $r^{2}$ results of 0.9646 (9-hour cure) and 0.8385 (11/2-hour cure).

\begin{tabular}{|c|c|c|c|}
\hline \multicolumn{2}{|c|}{ Table 1. Slope test results in the incisal area. } \\
$\begin{array}{c}\text { Slope Test } \\
\text { (Goodness of Fit) }\end{array}$ & $\begin{array}{c}\text { Hydrated } \\
\text { (Control) }\end{array}$ & $\begin{array}{c}\text { Dry-stored } \\
\text { (9-Hour Cure) }\end{array}$ & $\begin{array}{c}\text { Dry-stored } \\
\text { (1 1/2-hour Cure) }\end{array}$ \\
\hline $\mathbf{r}^{2}$ & 0.001452 & 0.9646 & 0.8385 \\
\hline P value & 0.9515 & 0.0029 & 0.0290 \\
\hline Deviation from zero & Not Significant & Significant & Significant \\
\hline
\end{tabular}

The slope test further revealed that the slopes for the dry-stored APD bases were significantly different $(p<0.05)$. There were no significant differences $(p>0.05)$ for the wet-stored APD bases. Figure 5 , which is a time series plot of the measure of deformation immediately after fabrication (Day 1) to each storage interval, further supports this. The F-test used to determine if the slopes were equal showed no significant difference $(p=0.51)$. The F-test for elevation testing $(p<0.05)$ also revealed no significant differences $(p=0.42)$.

\section{Deepest part of the midline posteriorly}

As presented in Table 2, the slope test results of the deepest part of the midline area of the APD bases posteriorly revealed high $r^{2}$ results, namely, 0.9919 (wetstored 9-hour cure), 0.6852 (dry-stored 9-hour cure) and 0.7730 (dry-stored 11/2-hour cure).

\begin{tabular}{|c|c|c|c|}
\hline $\begin{array}{c}\text { Slope Test } \\
\text { (Goodness of Fit) }\end{array}$ & $\begin{array}{l}\text { Hydrated } \\
\text { (Control) }\end{array}$ & $\begin{array}{l}\text { Dry-stored } \\
\text { (9-Hour Cure) }\end{array}$ & $\begin{array}{c}\text { Dry-stored } \\
\left(1^{1 / 2-h o u r ~ C u r e) ~}\right.\end{array}$ \\
\hline $\mathbf{r}^{2}$ & 0.9919 & 0.6852 & 0.7730 \\
\hline$P$ value & 0.0003 & 0.0836 & 0.0495 \\
\hline Deviation from zero & Significant & Not Significant & Significant \\
\hline
\end{tabular}

There were significant differences in the wet-stored 9 -hour cure and the dry-stored 11/2-hour cure $(p<0.05)$. Figure 6 further supports this. Overall, the F-test used to determine if the slopes were equal showed no significant difference $(p=0.15)$. A point that deserves to be mentioned is that the F-test for elevation testing ( $y$ intercepts) showed significant differences across the three groups $(p=0.00)$. This indicates that deformation across the three groups are similar and increased over time.

\section{Area of the maxillary right first molar}

As shown in Table 3, the slope test results of the APD bases in the maxillary right first molar area reveal- 


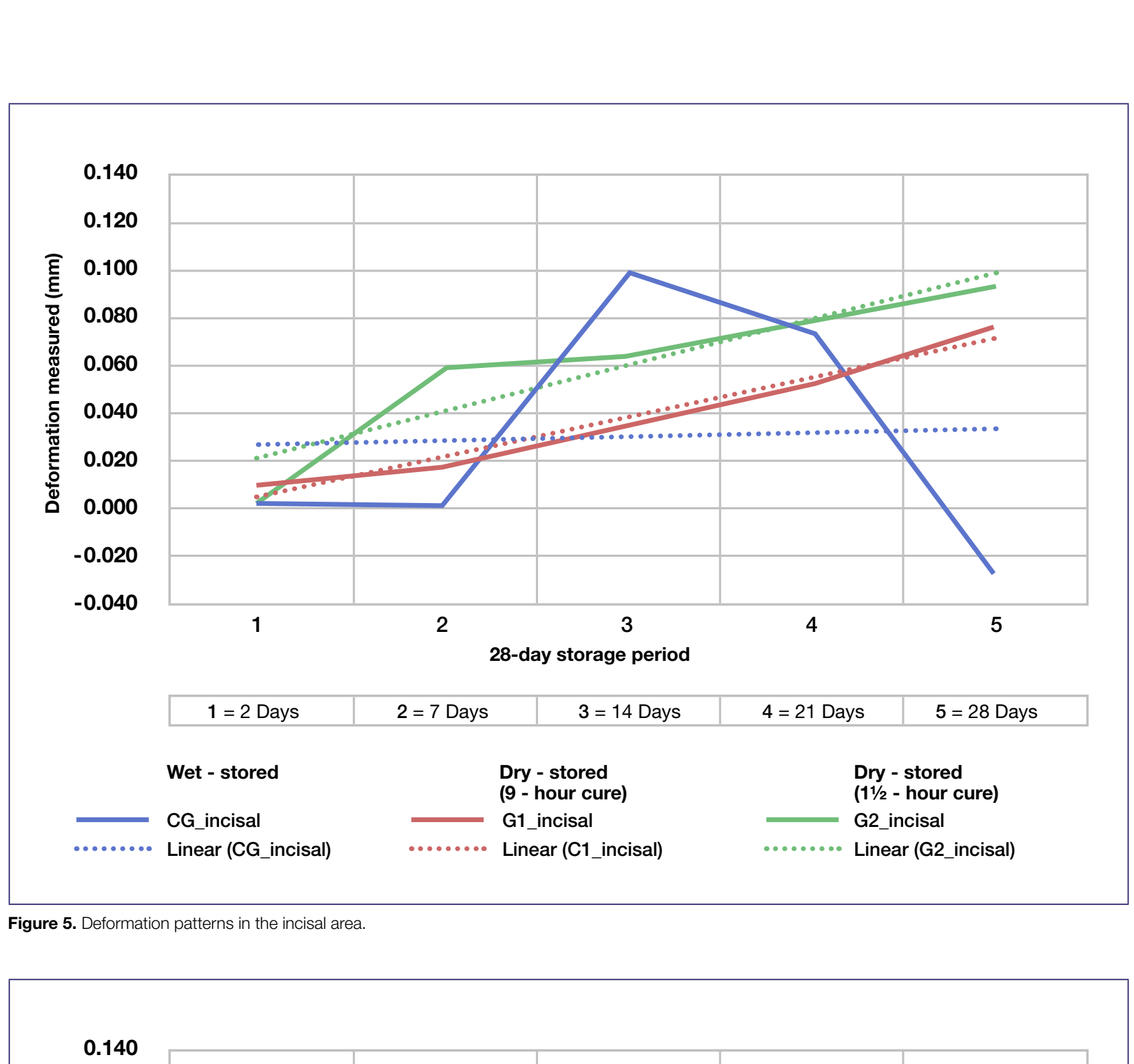

Figure 5. Deformation patterns in the incisal area.
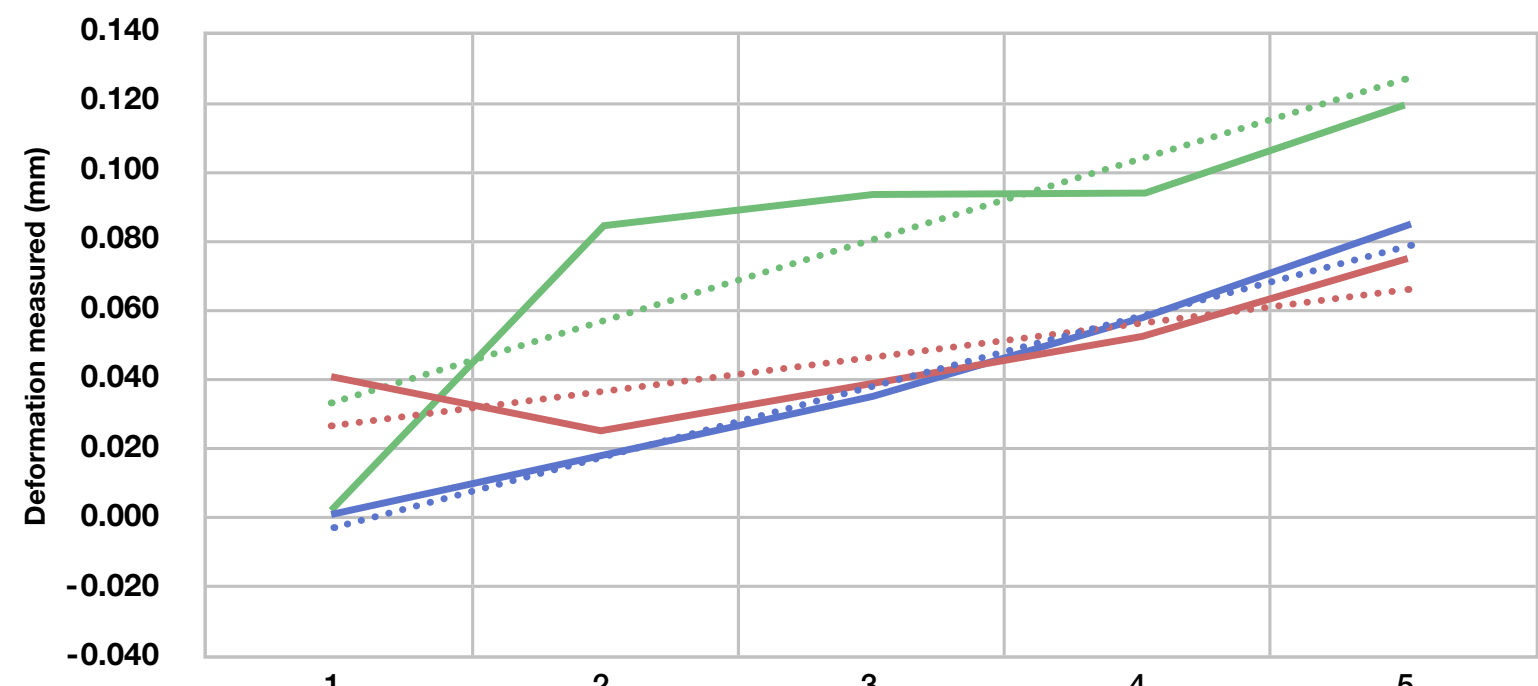

1

2

3

4

5

28-day storage period

\begin{tabular}{|l|l|l|l|c|}
\hline $\mathbf{1}=2$ Days & $2=7$ Days & $3=14$ Days & $4=21$ Days & $5=28$ Days \\
\hline
\end{tabular}

Wet - stored

CG_midline

Linear (CG_midline)
Dry - stored

(9 - hour cure)

G1_midline

Linear (C1_midline)
Dry - stored

(11/2 - hour cure)

G2_midline

Linear (G2_midline) 


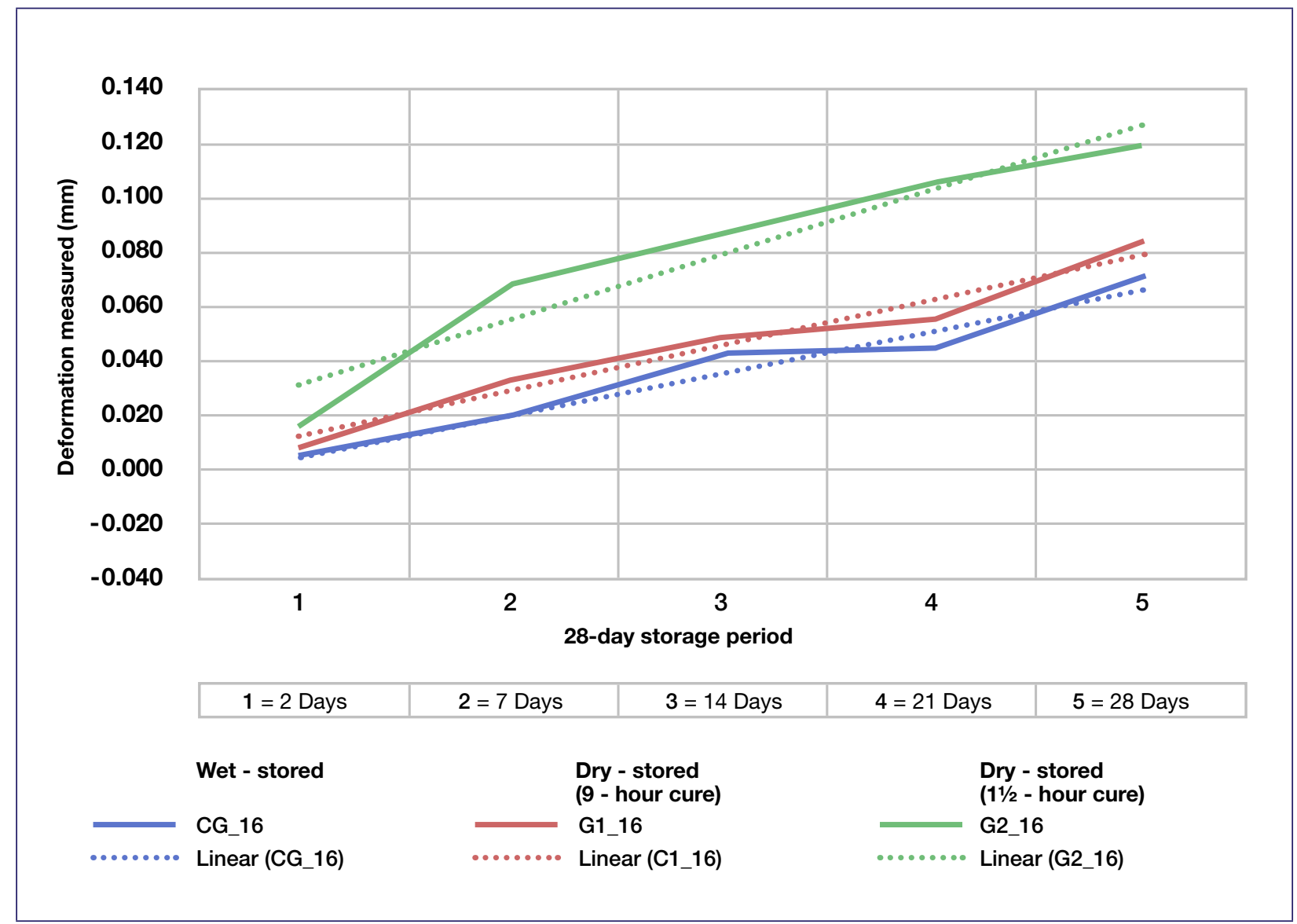

Figure 7. Deformation patterns in the maxillary right first molar area.

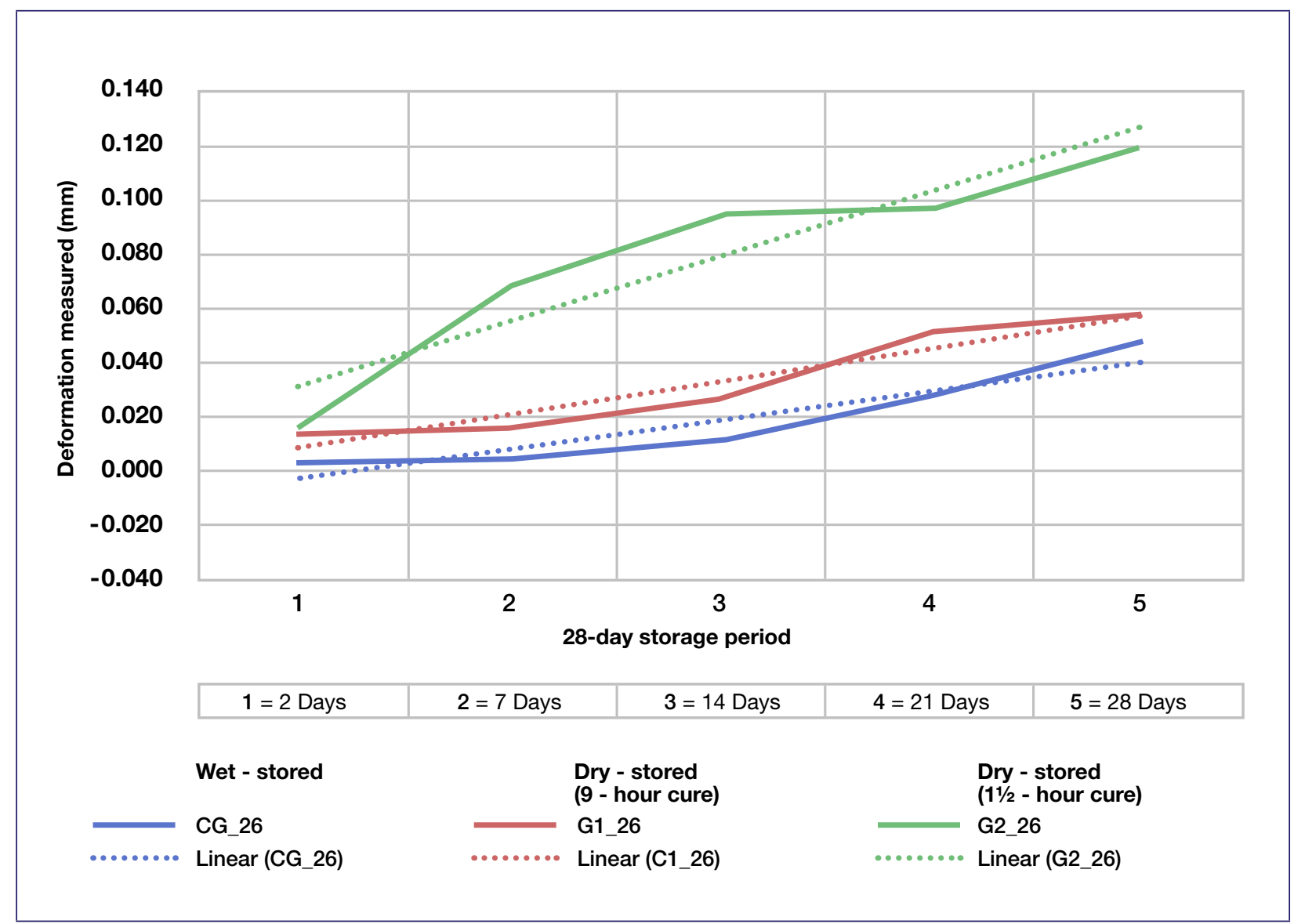

Figure 8. Deformation patterns in the maxillary left first molar area. 
ed high $r^{2}$ values of 0.9507 (wet-stored 9-hour cure), 0.9654 (dry-stored 9-hour cure) and 0.9167 (dry-stored

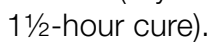

\begin{tabular}{|c|c|c|c|}
\hline \multicolumn{3}{|c|}{ Table 3. Slope test results in the maxillary right first molar area. } \\
$\begin{array}{c}\text { Slope Test } \\
\text { (Goodness of Fit) }\end{array}$ & $\begin{array}{c}\text { Hydrated } \\
\text { (Control) }\end{array}$ & $\begin{array}{c}\text { Dry-stored } \\
\text { (9-Hour Cure) }\end{array}$ & $\begin{array}{c}\text { Dry-stored } \\
\text { (1 1 1/2-hour Cure) }\end{array}$ \\
\hline $\mathbf{r}^{2}$ & 0.9507 & 0.9654 & 0.9167 \\
\hline P value & 0.0047 & 0.0028 & 0.0105 \\
\hline Deviation from zero & Significant & Significant & Significant \\
\hline
\end{tabular}

As illustrated in Figure 7, the slope test results further indicated that the slopes for all three groups were significantly different $(p<0.05)$.

From Figure 7, the slope pattern for the dry-stored (11/2-hour cure) APD bases is higher than the wet-stored and dry-stored (9-hour cure) APD bases. The slope pattern for the wet-stored 9-hour cure APD bases is the lowest from the three groups. Although the F-test used to determine if the slopes were equal showed no significant difference $(p=0.16)$, the F-test for elevation testing revealed significant differences across the three groups $(p=0.00)$.

\section{Area of the maxillary left first molar}

Table 4 shows that the slope test results of the APD bases in the maxillary left first molar area had high $r^{2}$ values of 0.8871 (wet-stored 9-hour cure), 0.9312 (dry-stored 9-hour cure) and 0.8891 (dry-stored 11/2hour cure). The slope test revealed significant differences across all groups.

\begin{tabular}{|c|c|c|c|}
\hline \multicolumn{3}{|c|}{ Table 4. Slope test results in the maxillary left first molar area. } \\
$\begin{array}{c}\text { Slope Test } \\
\text { (Goodness of Fit) }\end{array}$ & $\begin{array}{c}\text { Hydrated } \\
\text { (Control) }\end{array}$ & $\begin{array}{c}\text { Dry-stored } \\
\text { (9-Hour Cure) }\end{array}$ & $\begin{array}{c}\text { Dry-stored } \\
(11 / 2 \text {-hour Cure) }\end{array}$ \\
\hline Y-intercept & $-0.0140 \pm$ & $-0.003600 \pm$ & $0.008500 \pm$ \\
when $\mathbf{X}=0.0$ & 0.007514 & 0.006454 & 0.01602 \\
\hline $\mathbf{r}^{2}$ & 0.8871 & 0.9312 & 0.8891 \\
\hline P value & 0.0167 & 0.0078 & 0.0162 \\
\hline Deviation from zero & Significant & Significant & Significant
\end{tabular}

This is further supported by Figure 8, which shows a positive linear relationship for the wet-stored, dry-stored (9-hour cure) and dry-stored (11/2-hour cure) APD bases. The slope pattern for the dry-stored (11/2-hour cure) APD base is higher than the wet-stored and drystored (9-hour cure) APD bases.

The F-test used to determine whether the slopes were equal showed significant differences between the slopes $(p=0.04)$. Table 4 further shows that the dry-stored (11/2-hour cure) APD bases had the highest elevation (0.008500 \pm 0.01602$)$. The wet-stored APD bases, by contrast, had the lowest elevation $(-0.0140 \pm 0.007514)$.

\section{DISCUSSION}

The present study examined the effects of dry- and wet-storage conditions on the dimensional fit of APD bases. As seen in Figure 5, the slope pattern of the wet-stored APD bases increases from days 2-14 and decreases from days 14-28. The irregular slope pat- tern could be attributed to the affinity of APD bases absorbing water and subsequently expanding.

A critical point that deserves to be mentioned is that within the context of this research expansion of the APD base is a measure of a decrease in the gap $(\mathrm{mm})$ between the APD base and maxillary cast. Conversely, contraction is the measure of an increase in the gap $(\mathrm{mm})$ between the APD base and maxillary cast.

It can therefore be inferred that expansion of the APD bases occurred from days 14-28. This is consistent with the study conducted by Rimple, Gupta, Kamra et al. ${ }^{13}$ and Hamouda, El-Sharkawi, Gomaa et al. ${ }^{14}$

They showed that the highest expansion of complete dentures stored in water occurred in the first month of the 3-month storage period. Another noteworthy point is that the dry-stored 9-hour cure APD bases in this study also showed visible changes in dimensional stability across all reference areas.

The results of this study supports the work of Miessi, Goaito, Santos et al. ${ }^{7}$ as the loss of water and incomplete polymerisation affects the dimensional fit of APD bases, especially when the dentures are dry stored.

In contrast to Lim and Lee, ${ }^{3}$ who reported that the degree of deformation over a one month dry storage period was low, the results of this study showed that the highest deformation occurred on day 28 of the wet- and dry-storage periods. In particular, the deformation across the four reference areas was higher for the dry-stored 11/2-hour cure than the wet-stored and dry-stored 9-hour cure APD bases.

The wet-stored 9-hour cure APD bases had the lowest deformation. This corroborates with the advice given by Anusavice, Shen and Rawls ${ }^{8}$ that a 9-hour polymerisation cycle helps to reduce polymerisation shrinkage thereby achieving dimensionally stable denture bases. This must be considered in conjunction with the recommendation given by several other authors ${ }^{15-18}$ that dentures are to be stored in water for at least 24-hours post manufacture in order to compensate for polymerisation shrinkage.

While the results of the slope test for the APD bases revealed no significant difference incisally, there were significant differences posteriorly. This is consistent with the findings of Lim and Lee, ${ }^{3}$ Consani, Domitti and Consani $i^{15}$ and Arora, Khindaria, Garg et al. ${ }^{18}$, who showed deformation in the posterior palatal areas of maxillary complete dentures during dry-storage. The hypothesis tested that is there is a significant relationship between dry-storage conditions and deformation of acrylic partial denture bases was therefore accepted.

The prominent features of this study showed that low humidity affected the posterior areas of the APD bases on dry-storage, specifically confirming the highest deformation recorded was in the maxillary first molar areas.

This study conclusively showed that the deformation patterns of APDs were reduced if kept hydrated on storage. 


\section{Limitation}

Although the silicone wafer method provided valuable baseline data on the deformation of APDs, the integrity of the silicon wafer could have been affected during the measuring of mis-fits over the 28-days storage period. Future studies should therefore consider using advanced technology such as surface matching software and 3D scanners for scanning impressions and casts.

\section{Acknowledgements}

This study was supported by The National Research Foundation (NRF), Durban University of Technology, grant \#UID9875. The authors further thank Mr Deepak Singh and Ms Denise Angela Skea for their invaluable contributions made to this study.

\section{References}

1. Consani RLX, Monterio VL, Mesquita MF, Consani S. The influence of storage on dimensional changes in maxillary acrylic denture bases and the effect on tooth displacement. European J Prosthet Dent. 2011; 19: 105-10.

2. Kasina PS, Ajaz T, Attili S, Surapaneni H, Cherukuri M, Srinath HP. To evaluate and compare the porosities in the acrylic mandibular denture bases processed by two different polymerisation techniques, using two different brands of commercially available denture base resins - an in vitro study. J Int Oral Health. 2013; 6: 72-7.

3. Lim SR, Lee JS. Three dimensional deformation of drystored complete denture base at room temperature. J Adv Prosthodont 2016; 8: 296-303.

4. Joshi NP, Sanghvi SJ. Water sorption by maxillary acrylic resin denture base and consequent changes in vertical dimension. J Pierre Fauchard Acad. 1994; 8: 97-106.

5. Al Nori AK, Hussain MA, Rejab LT. Water sorption of heat cured acrylic resin. Al-Rafidain Dent J. 2006; 7: 186-194.

6. Meloto, CB, Silva-Concilio LR, Machado C, Joia FA, Rizzatti-Barbosa CM. Water sorption of heat-polymerized acrylic resins processed in mono and Bimaxillary flasks. Braz Dent J. 2006; 17: 122- 5 .
7. Miessi AC, Goiato MC, Santos DM, Dekon SF, Okidi RC. Influence of storage period and effect of different brands of acrylic resin on the dimensional accuracy of the maxillary denture base. Braz Dent J. 2008; 19: 204-8.

8. Anusavice KJ, Shen C, Rawls HR. Phillips Science of Dental Materials. 12 ${ }^{\text {th }}$ ed. St. Louis: Elsevier; 2013. $745-50$.

9. Ristic B, Carr L. Water sorption by denture acrylic resin and consequent changes in vertical dimension. J Prosthet Dent. 1987: 58: 689-93.

10. Weatherbase. Kimberley, South Africa Travel Weather Averages (Weatherbase) [Internet]. Weatherbase. [cited 2015 Nov5]. Available from: http://www.weatherbase.com/weather/ weather. php3?s $=83486$ \& cityname $=$ Kimberley-Northern-Cape-South-Africa.

11. Ghani, F., Kikuchi, M., Lynch C. D. and Watanabe, M. Effect of some curing methods on acrylic maxillary denture base fit. Eur J Prosthodont Restor Dent. 2010; 18: 132-8.

12. Motulsky HJ. Advantages of using Prism's nonlinear regression analysis to fit straight lines [Internet]. GraphPad. [cited 2020Mar6]. Available from: https://www.graphpad.com/ support/faq/advantages-of-using-prisms-nonlinear-regression-analysis-to-fit-straight-lines/.

13. Rimple, Gupta A, Kamra M, Balagopal. An evaluation of the effect of water sorption on dimensional stability of the acrylic resin denture bases. Int $\mathrm{J}$ Contemp Dent. 2011; 2: 43-7.

14. Hamouda IM., El-Sharkawi AN, Gomaa AM, El Sharkawi, NE. Effect of arch form and water sorption on the palatal base adaption of ProBase hot versus the conventional heat cured acrylic resin. Int J Dent Oral Sci. 2016; 3: 193-9.

15. Consani RLX, Domitti, SS, Consani S. Effect of a new tension system, used in acrylic resin flasking, on the dimensional stability of denture bases. J Prosthet Dent. 2002; 88: $285-9$.

16. Consani RLX, Mesquita MF, Sinhoreti MAC, Consani $S$ Influence of the deflasking delay time on the displacements of maxillary denture teeth. J Appl Oral Sci. 2003; 11: 2-8.

17. Kobayashi N, Komiyama O, Kawara M. Reduction of shrinkage on heat-activated acrylic denture base resin obtaining gradual cooling after processing. J Oral Rehabil. 2004; 31: $710-6$.

18. Arora SS, Khindaria K, Garg S, Mittal S. Comparative evaluation of linear dimensional changes of four commercially available heat cure acrylic resins. Contemp Clin Dent. 2011; 2: 82-7.

\section{Do the CPD questionnaire on page 104}

The Continuous Professional Development (CPD) section provides for twenty general questions and five ethics questions. The section provides members with a valuable source of CPD points whilst also achieving the objective of CPD, to assure continuing education. The importance of continuing professional development should not be underestimated, it is a career-long obligation for practicing professionals.

Online CPD in 6 Easy Steps

1 Go to the SADA website www.sada.co.za.

2 Log into the 'member only' section with your unique SADA username and password.

3 Select the CPD navigation tab.

4 Select the questionnaire that you wish to complete.

5 Enter your multiple choice answers. Please note that you have two attempts to obtain at least $70 \%$.

6 View and print your CPD certificate. 